\title{
Chaos in a Feedback Fabry-Perot Interferometer
}

\author{
Toshiyuki Takizawa, Yun Liu, and Junji Ohtsubo
}

\begin{abstract}
A novel hybrid chaotic system composed of a laser diode source and a Fabry-Perot interferometer with an electrical feedback is proposed. The delayed-differential equation describing the system input-output relation is derived and studied by numerical simulations. The instability of the output power from the laser diode is investigated experimentally and its behavior is proved to be chaotic. The comparison between the theoretical analysis and the experimental results shows good agreement.
\end{abstract}

\section{INTRODUCTION}

QINCE Ikeda et al. predicted chaotic behavior in a ring $\boldsymbol{N}$ cavity [1], the dynamical behaviors of optoelectronic hybrid systems with delayed feedback have been studied and demonstrated experimentally [2], [3]. In spite of much effort for investigating optical or hybrid chaos, routes to chaos have not been completely understood yet. Recently, Liu and Ohtsubo have demonstrated chaotic behavior in a system which consists of a laser diode Twyman-Green interferometer with an electronic delayed feedback loop [3]. In all of these systems, a time delay that is larger than the intrinsic relaxation time or the response time of the system is introduced into the feedback loop. The dynamics of such a system can be described by a delayed-differential equation and its behavior can be theoretically studied based on this equation [2]-[7]. Such a hybrid system is convenient for investigating the chaotic behavior because of its experimental simplicity and easiness of the parameter control. It is also suitable for the observation of the Ikeda's instability which requires the response time of the system being short enough to allow a period- $2 \tau_{r}$ oscillation ( $\tau_{r}$ being the delay time of the system). We have proposed a new chaotic system which consists of a laser diode and a Fabry-Perot interferometer with an electrical delayed feedback loop [8]. The chaotic behavior of the laser output power has been experimentally observed.

In this paper, the theoretical derivation of the relation between the bias injection current and the laser output power in the feedback Fabry-Perot interferometer is given. Investigation of the bifurcation routes to chaos is conducted theoretically and experimentally. In the proposed system, the nonlinearity of the system is produced by the nonlinear relation of the transmitted power with the laser oscillation frequency. The output power from the Fabry-Perot resonator is fed back to the injection current of the laser diode source with an

Manuscript received July 27, 1992; revised April 19, 1993. This work was supported by the CASIO Science Promotion Foundation.

T. Takizawa and J. Ohtsubo are with the Faculty of Engineering, Shizuoka University, Hamamatsu, 432 Japan.

Y. Liu is with the Graduate School of Electronic Science and Technology, Shizuoka University, Hamamatsu, 432 Japan.

IEEE Log Number 9215259.

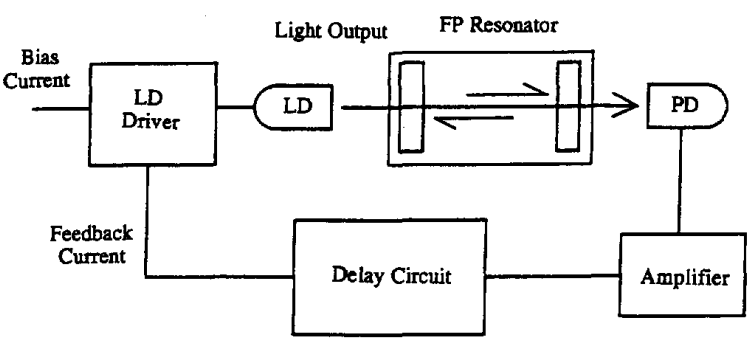

Fig. 1. Experimental setup. LD: laser diode, PD: photodiode, and FP: Fabry-Perot.

electronic delayed circuit. The injection current change induces the oscillation frequency change, thus closing the loop and forming the nonlinear relation between the laser output powers before and after the time delay. The chaotic behavior is experimentally observed in the laser output power. We also derive the delayed-differential equation which describes the output power relations of the system. The computer simulation is done by using the derived delayed-differential equation. The comparison between the experimental results and theoretical predictions shows good agreement.

\section{THEORY}

The experimental model is shown in Fig. 1. The light from a laser diode (LD) passes through a Fabry-Perot (FP) resonator and its output power is detected by a photodetector (PD). The detected signal is amplified and fed back into the injection current with an appropriate delay time. Due to the modulation property of the laser diode, the output power $P_{o}$ has a linear relation with the feedback injection current $I_{f}$ and is written by

$$
P_{o}=\alpha\left(I_{f}+I_{b}-I_{t h}\right)
$$

where $\alpha$ is the conversion coefficient of the injection current to the output power, and $I_{b}$ and $I_{t h}$ are the bias and threshold currents, respectively. The oscillation frequency $f$ is related to the output power as

$$
f=f_{0}-\frac{\chi}{\alpha} P_{o}
$$

where $f_{0}$ is the oscillation frequency at the threshold injection current $I_{t h}$ and $\chi$ is the conversion coefficient of the injection current to the oscillation frequency.

Next, we consider the spectral profile of the emitted light from the laser diode. We assume that the spectral profile of 
the laser diode has a Lorentzian line shape and is given by

$$
P_{L D}=\frac{\beta P_{o}}{1+\left(\frac{\nu-f}{\Delta \nu}\right)^{2}}
$$

where $\beta$ is the normalization coefficient, $\nu$ is the frequency, and $\Delta \nu$ is the half width at half maximum (HWHM) of the spectral profile. If the reflectance of the mirrors in the Fabry-Perot resonator is nearly unity and the width of the transmission function is much narrower than the spectral profile of the laser oscillation (i.e., the assumption of the high finesse of the Fabry-Perot etalon), the transmittance function $\gamma$ of the Fabry-Perot resonator can be assumed to be a sequence of delta functions

$$
\gamma=\sum_{m=0}^{\infty} \delta_{v, m} \nu_{f}
$$

where $\delta$ is a Kronecker delta-function, and $\nu_{f}$ is the mode separation of the Fabry-Perot resonator. Using (3) and (4), the transmitted power from the Fabry-Perot resonator is

$$
P_{x}=\beta P_{o} \sum_{m=0}^{\infty} \frac{1}{1+\left(\frac{m \nu_{f}-f_{0}+\chi P_{o} / \alpha}{\Delta \nu}\right)^{2}} .
$$

Consequently, the feedback injection current $I_{f}$ is given by

$$
I_{f}=-\mu^{\prime} P_{x}
$$

where $-\mu^{\prime}$ is the feedback gain and the minus sign implies the negative feedback of the loop. Substituting (5) and (6) into (1), we finally obtain the fundamental equation of the feedback loop as

$$
P=P_{b}-\mu P_{o} \sum_{m=0}^{\infty} \frac{1}{1+\left(\kappa P_{o}-\xi_{m}\right)^{2}}
$$

where $P$ is the laser power after the feedback, $P_{b}$ is the bias power and equals to $\alpha\left(I_{b}-I_{t h}\right), \mu$ is the total feedback coefficient and is given by $\mu^{\prime} \alpha \beta, \kappa=\chi / \alpha \Delta \nu$, and $\xi_{m}=$ $\left(f_{0}-m \nu_{f}\right) / \Delta \nu$. The output power is expressed as the infinite summation over the integers $m$, but in the actual situation, only a few modes corresponding to certain integer values of $m$ contribute to the summation.

Taking the continuous property of the actual system into consideration, the laser output power is written by the following delayed-differential equation as

$$
T_{m} \frac{d P_{o}(t)}{d t}+P_{o}(t)=F\left\{P_{o}\left(t-\tau_{r}\right)\right\}
$$

where $F(P)$ represents the function of the right-hand side in (7), and $T_{m}$ and $\tau_{r}$ are the response time of the system and the delay time in the feedback loop, respectively. For a delay time larger than the system response, i.e., $T_{m} \ll \tau_{r}$, the differential term in (8) is neglected and the delayed-differential equation is simplified as a difference equation

$$
X_{n+1}=F\left(X_{n}\right)
$$

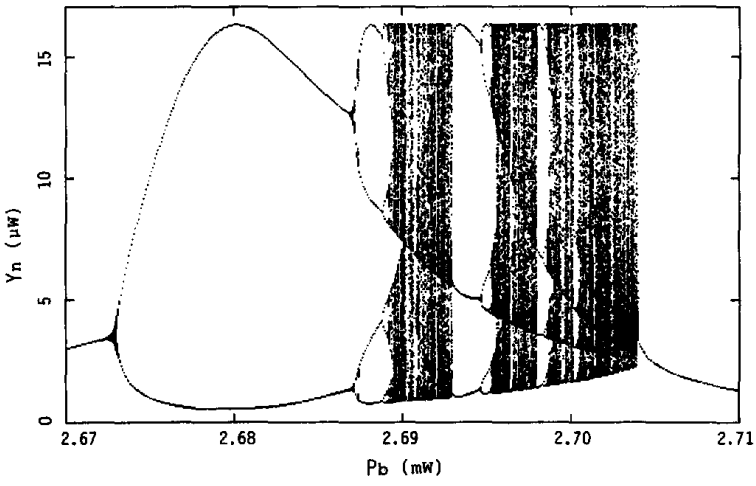

Fig. 2. Bifurcation diagram for the bias power from $P_{b}=2.67$ to $2.71 \mathrm{~mW}$ under the conditions of $\mu=0.017$ and $\kappa=122.94 \mathrm{~mW}^{-1}$.

where $X_{n}=P_{o}\left(t_{0}+n \tau_{r}\right)$ ( $n$ being an integer number and $t_{0}$ being a certain initial time). The transmitted power from the Fabry-Perot resonator at the discrete system is given by

$$
Y_{n}=\beta X_{n} \sum_{m=0}^{\infty} \frac{1}{1+\left(\kappa X_{n}-\xi_{m}\right)^{2}} .
$$

In the following theoretical and experimental discussions, the output power $Y_{n}$ from the Fabry-Perot resonator is investigated in relation to the laser output power $X_{n}$. Although the transmitted power from the Fabry-Perot resonator is not exactly equal to the laser output power itself, the chaotic behavior of the transmitted power can be considered to be equivalent to that of the laser output power.

In the present system, the bias output power $P_{b}$ can be varied arbitrarily. Fig. 2 shows the calculated bifurcation diagram of the output power against the bias output power for the range from $P_{b}=2.67$ to $2.71 \mathrm{~mW}$ at the fixed values of $\mu=0.017$ and $\kappa=122.94 \mathrm{~mW}^{-1}$. The parameter values are compatible with those in the experiment as discussed in later. In Fig. 2, the stationary state at a low output power of the bias current evolves into the period-doubling bifurcation routes and finally results in the chaotic state. In the chaotic region, we can observe the period-three window in this diagram. The diagram is very helpful for investigating the period-doubling bifurcation routes to chaos.

Since the bifurcation diagram is calculated by the difference equation (9) and the response time of the system is neglected, it is difficult to investigate the dynamical behavior of the laser output power from the difference equation. To explain the dynamical behavior of the system's output, we must consider the continuity of the actual system. The differential term in (8) is linearly approximated as

$$
\frac{d P_{o}(t)}{d t} \approx \frac{P_{o}(t+\Delta t)-P_{o}(t)}{\Delta t}
$$

where $\Delta t$ is the time interval which is small enough to realize this approximation. The time interval $\Delta t$ can be recognized as the interval of the transition of the system's output. Using this approximation, the dynamical behavior of the laser output was investigated by the simulation under the conditions of $\tau_{r}=1.00 \mathrm{~ms}, T_{m}=0.086 \mathrm{~ms}$, and $\Delta t=1 \mu \mathrm{s}$, where the 

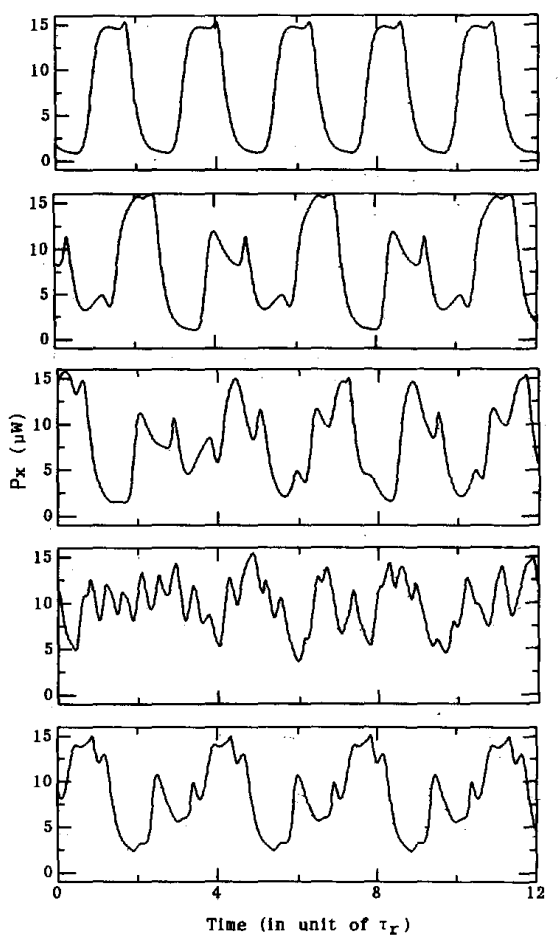

Fig. 3. Time series of the output power from the Fabry-Perot resonator. From top to bottom: (a) Period-two state at $P_{b}=2.684 \mathrm{~mW}$. (b) Period-four state at $P_{b}=2.690 \mathrm{~mW}$. (c) Prechaotic state at $P_{b}=2.692 \mathrm{~mW}$. (d) Completely chaotic state at $P_{b}=2.702 \mathrm{~mW}$. (e) Period-three state at $P_{b}=2.698 \mathrm{~mW}$.

values of $\tau_{r}$ and $T_{m}$ were almost the same as those for the experiment.

The bifurcation scenario can be observed in several discrete bias output power regions. Fig. 3 shows the results of the simulation for a bias output power region corresponding to the bifurcation diagram plotted in Fig. 2. As predicted in Fig. 2 for the bias output power below a certain critical value of $0.2673 \mathrm{~mW}$, the system remains stable, whereas, beyond this critical value, the light output evolves into unstable states. Fig 3(a) is the time series after the system becomes unstable at the first point at which the bias current exceeds a certain critical bias point. The series has the period $2 T$ which is slightly larger than twice the delay time. The discrepancy between the time $T$ and the delay time $\tau_{r}$ is attributed to the response time of the system. The time interval $T$ is calculated as $1.10 \mathrm{~ms}$. The difference $0.10 \mathrm{~ms}$ is almost the same as the response time of the system. With a slight increase of the bias power, the period-doubling transition to the next state occurs, and the output power conforms to a period-four solution, as shown in Fig. 3(b). Fig. 3(c) is prechaotic state and so-called period-two chaos. With further increase of the bias power, the perioddoubling bifurcation routes get into a complete chaos. It is noted that the waveforms, except for the period-two state, contain many spikes due to the effects of the differential term. At the chaotic state, the output power irregularly changes even within the delay time. In the chaotic region, we obtained the
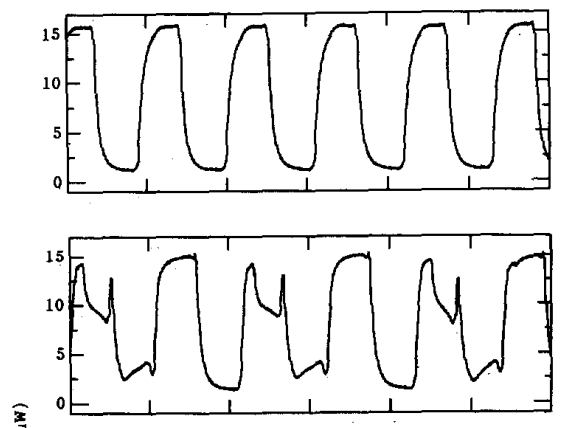

胥
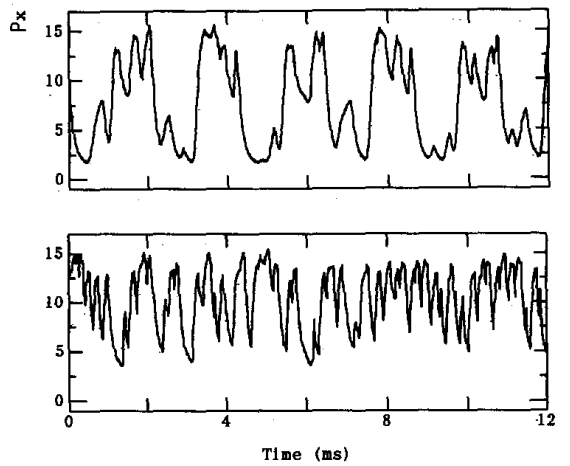

Fig. 4. Experimental results of the output power under the conditions of $\tau_{r}=1 \mathrm{~ms}, \mu=0.017$. From top to bottom: (a) Period-two state at $P_{b}=2.546 \mathrm{~mW}$. (b) Period-four state at $P_{b}=2.558 \mathrm{~mW}$. (c) Prechaotic state at $P_{b}=2.571 \mathrm{~mW}$. (d) Completely chaotic state at $P_{b}=2.620 \mathrm{~mW}$.

period-three solution as shown in Fig. 3(e). The period of the waveform is about $3.4 \tau_{r}$.

\section{EXPERIMENTS AND RESULTS}

In the experimental setup shown in Fig. 1, the light source was a single mode CSP laser diode of Hitachi HL $7801 \mathrm{E}$ which oscillated at a wavelength of $0.780 \mu \mathrm{m}$ and a maximum power of $5 \mathrm{~mW}$. The spectral width of the laser diode was measured to be $\Delta \nu=0.2 \mathrm{GHz}$ at $I_{b}=50.0 \mathrm{~mA}$ (the threshold current was $I_{t h}=35.0 \mathrm{~mA}$ ). The conversion coefficient $\chi$ was measured to be $3.1 \mathrm{GHz} / \mathrm{mA}$. The finesse of a Fabry-Perot resonator was 200 . The value is large enough to assume a delta-function-like transmission of the resonator as is discussed in (4). The free spectral range of the Fabry-Perot resonator was $7.5 \mathrm{GHz}$. The light from the laser diode was transmitted through the Fabry-Perot resonator and detected by a PIN photodiode. The detected signal was amplified and passed through a digital delay circuit which consisted of a 12-bit first-in-first-out (FIFO) dual-port memory through an analogto-digital converter. After a delay time $\tau_{r}$, the digital signal was fed to a digital-to-analog converter and into the injection current of the laser diode. The time lag in the delay circuit was set within the range of $1 \mu \mathrm{s}$ to $4 \mathrm{~ms}$ under the control of a microcomputer.

Some experimental results of the output power from the Fabry-Perot for various bias powers $P_{b}$ at $\tau_{r}=1 \mathrm{~ms}$ and $\mu=0.017$ are shown in Fig. 4(a)-(d). In the experiment, 

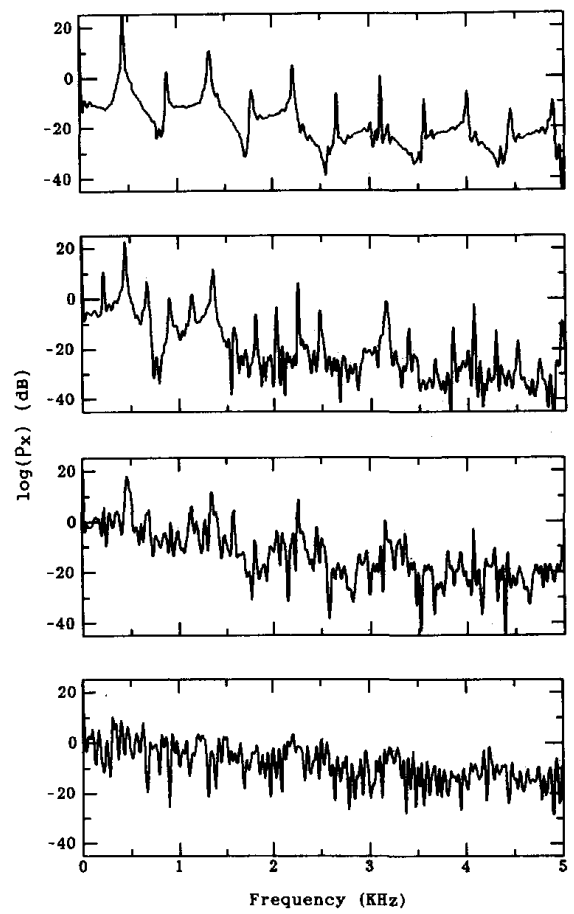

Fig. 5. Spectra of the output powers corresponding (top to bottom) to Fig. 4(a)-(d).

the bias currents to the laser diode were measured, but the corresponding bias output powers could be calculated by using the value of $0.123 \mathrm{~mW} / \mathrm{mA}$ of the conversion efficiency from the current to the laser power. Fig. 5(a)-(d) shows the power spectra of the output powers corresponding to Fig. 4. Fig. 4(a) is the time-dependent transmitted power from the Fabry-Perot resonator for the period-two solution. The period of the waveform is $2.20 \mathrm{~ms}$. The corresponding spectral peak can be recognized and also many subpeaks appear in the higher order harmonics in Fig. 5(a). With increase of the bias power, we observed the transition to the period-four state as shown in Fig. 4(b). In the corresponding power spectrum shown in Fig. 5(b), not only the main peak $f_{1}=1 / 2 T$ but also the subharmonic frequency $f_{1} / 2$ is observable. Fig. 4(c) shows the period-two chaos. Though the periodicity can be seen in the waveform, the waveforms of any two period durations are different from each other. The harmonic spectrum in Fig. $5(\mathrm{c})$ becomes broader compared with the periodic states. But we can clearly see the peak at $f_{1}$ which corresponds to the fundamental frequency. On the other hand, no obvious period is observable in the waveform in Fig. 4(d) and a clear spectral peak also can not be seen in the corresponding spectrum in Fig. 5(d). The output power corresponds to the chaotic state. The evolution to chaos in the experiments is almost the same as the theoretical predictions. The parameter values in the simulation in Fig. 3 were compatible with those of the experiment.

We have experimentally observed the existence of the period-three state within the chaotic region. Fig. 6(a) shows the time series of the output from the Fabry-Perot resonator

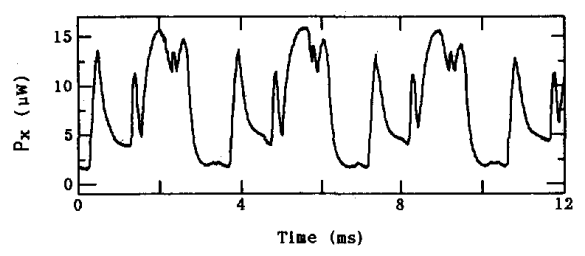

(a)

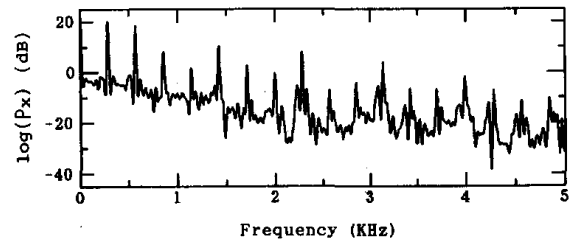

(b)

Fig. 6. Experimental results of period-three state under the same conditions of Fig. 4. (a) Waveform at $P_{b}=2.583 \mathrm{~mW}$ and (b) corresponding power spectrum.

and Fig. 6(b) is the corresponding power spectrum. The delay time and the response time of the system were the same as in the previous results in Fig. 4. The period of the time series was calculated as $3.40 \mathrm{~ms}$, which is the same as the numerical result. The existence of the period-three state is very important in the chaos experiment. It can be considered as evidence of deterministic chaos for our system.

Figure 7 shows the phase portrait of the output power from the Fabry-Perot resonator for various bias output powers of the laser diode. Fig. 7(a)-(d) are the portraits of Fig. 4(a)-(d) and Fig. 7(e) is that of Fig. 6(a). The portraits were plotted by differentiating the output powers with an appropriate low-pass filter to avoid the influence of high frequency noises. In Fig. 7 (a)-(d), the orbits evolve into the period-doubling bifurcation routes as they bury inside the loop of the period-two state. The collecting points of the maps on the axis $d P_{X} / d t=0$ correspond to the equilibrium states of the output power. The orbits on the period-two and period-four states contain two and four collecting points, respectively. On the contrary, the orbit at completely chaotic state in Fig. 7(d) has no collecting point on the map. The orbit of the period-three state shown in Fig. 7(e) has three collecting points and is similar to the map of a Möbius strip.

\section{CONClusion}

We have proposed a new system for an optoelectronic hybrid chaotic system which consists of a laser diode, a Fabry-Perot resonator, and an electronic feedback circuit. The theoretical investigation has been made by using a delayed-differential equation which describes the system. The experimental results have been supported by the theoretical predictions. We have observed the period-three solution in the output power from the Fabry-Perot resonator. The observations of the periodthree solution together with the experimental investigations for the period-doubling bifurcation imply the existence of deterministic chaos in the laser output power. 


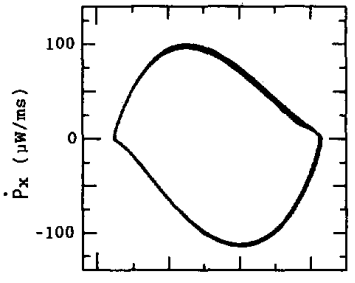

(a)

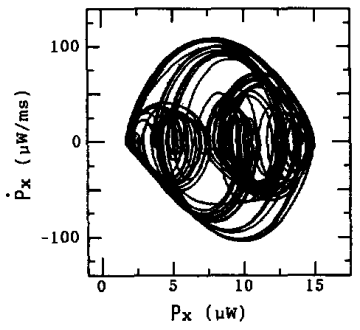

(c)

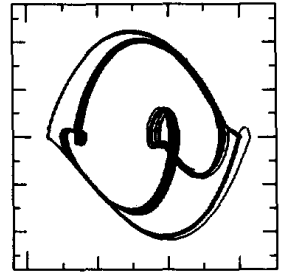

(b)

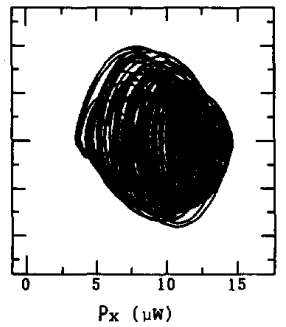

(d)

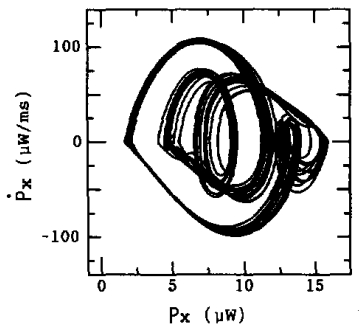

(e)

Fig. 7. Phase portraits of the output powers obtained from Fig. 4 and Fig. 6 . (a) Period-two state. (b) Period-four state. (c) Prechaotic state. (d) Completely chaotic state. (e) Period-three state.

In this paper, we have only discussed the fundamental period-doubling bifurcation scheme. However, higher harmonic bifurcations in the output power are expected at an appropriate delay time. Therefore, further investigations are required for the present chaotic system. Finally, we point out that the same phenomena may be observed in an intrinsic optical feedback system. With an optical feedback of the interfered signal to the laser diode cavity, we can realize a fast optical oscillation of more than a gigahertz by using, for example, the period-two solution in the laser output. But, in this case, the response time or the relaxation of the carrier density in a laser diode cavity may become an important factor.

\section{REFERENCES}

[1] K. Ikeda, "Multiple-valued stationary state and its instability of the transmitted light by a ring cavity system," Opt. Commun., vol. 30 , pp. $257-261,1979$.

[2] F. A. Hopf, D. L. Kaplan, H. M. Gibbs, and R. L. Shoemaker, "Bifurcations to chaos in optical bistability," Phys. Rev. A, vol. 25, pp. 2172-2182, 1982.

[3] J. Ohtsubo and Y. Liu, "Optical instability and chaos in an active interferometer," in Optics in Complex Systems, F. Lanzl, H. Preuss, and G. Weigelt, Eds., Proc. Soc. Photo-Opt. Instrum. Eng., vol, 1319, pp. $50-51,1990$.

[4] Y. Liu and J. Ohtsubo, "Chaos in an active interferometer," J. Opt. Soc. Am. B, vol. 9, pp. 261-265, 1992.

[5] —_, "Observation of higher harmonic bifurcations in a chaotic system using a laser diode active interferometer," Opt. Commun., vol. 85, pp. 457-461, 1991.

[6] _-, "Dynamical behavior of an optical nonlinear system using a laser diode active interferometer," Rep. Grad. School Electron. Sci. Technol. Shizuoka Univ., vol. 12, pp. 23-28, 1992.

[7] —- "Period-three cycle in a chaotic system using a laser diode active interferometer," Opt. Commun., vol. 93, pp. 311-317, 1992.

[8] T. Takizawa, Y. Liu, and J. Ohtsubo, "Chaos in laser diode with electrical feedback from Fabry-Perot interferometer," in From Galileo's "Occhialino" to Optoelectronics, \} P. Mazzoldi, ed. Singapore: World Scientific, 1993, pp. 858-862.

Toshiyuki Takizawa received the B.S. degree in optoelectronics and mechanical engineering from Shizuoka University, Hamamatsu, Japan, in 1992. He is now a graduate student at the Tokyo Institute of Technology, where he is engaged in research on quantum-well semiconductor lasers.

$\mathrm{He}$ is a member of the Japanese Society of Applied Physics.

Yun Liu received the B.S. degree in precision mechanical engineering from University of Science and Technology of China in 1986 and the M.S. degree in optoelectronics and mechanical engineering from Shizuoka University, Hamamatsu, Japan, in 1991. Currently, he is pursuing the Ph.D. in the Graduate School of Electronic Science and Technology, Shizuoka University. His current research interests include bistability and chaos in laser systems. $\mathrm{He}$ is a member of OSA and the Japanese Society of Applied Physics.

Junji Ohtsubo received the B.S. degree in electronics from Kyusyu Institute of Technology, Japan, in 1973. He received the M.S. and Ph.D. degrees in electronics from Hokkaido University in 1975 and 1978, respectively.

In 1978, he joined the Mechanical Engineering Laboratory. During 1981-1982, he was a research associate at the Institute of Optics, University of Rochester. He joined Shizuoka University as an associate professor in 1985 and is presently a professor. His current research interests are statistical optics, speckle, optical inspections, active interferometry, optical information processing, and nonlinear dynamics in optics.

Prof. Ohtsubo is a Member of OSA, SPIE, the Japanese Society of Applied Physics, and the Laser Society of Japan. 